\title{
Self-measurement of blood pressure
}

\author{
WORLD HYPERTENSION LEAGUE ${ }^{1}$
}

\begin{abstract}
Although experience is still limited and more research is needed, the World Hypertension League recommends self-measurement of blood pressure in selected patients as an additional source of information to the practising physician, and as a way of encouraging patients to participate more actively in the therapeutic regimen.
\end{abstract}

Self-measurement of blood pressure-readings taken by lay persons on themselves or on a member of their family - is known to be spreading rapidly in a number of countries. Statements on the appropriate uses of such measurements have therefore been published by national bodies for hypertension control, such as the U.S. National High Blood Pressure Education Program (1) and the German League against Hypertension (2). An increasing variety of instruments are being designed and marketed for selfmeasurement and people are now more exposed to publicity on these devices than even in the recent past.

The adoption of self-measurement depends to a great extent on social, cultural, economic, and medical factors. Purchasing an instrument is outside the reach of most patients in a developing country; in some societies, the act of self-measurement may be unacceptable to the majority of the public, or to the majority of physicians. Nevertheless, in view of the increasing use of self-measurement and of the potential benefits for patients, the Council of the World Hypertension League felt that an analysis of the problem and a statement on the subject would be of benefit for present and future activities to control hypertension.

\footnotetext{
1 The World Hypertension League comprised, in 1987, the following 22 member organizations: National Heart Foundation of Australia; Belgium Hypertension Committee; Canadian Coalition against Hypertension; Danish Society of Hypertension; French National Committee against Arterial Hypertension; German League against Hypertension; Hypertension Committee of the Hungarian Society of Cardiology; All India Heart Foundation; Israel Hypertension Society; Italian League against Hypertension; Japan Heart Foundation; Hypertension League of Morocco; Polish Society of Hypertension; Portuguese League against Hypertension; Senegal Foundation for Hypertension and Heart Disease Control; Southern African Hypertension Society; Spanish League against Hypertension; Swedish League against Hypertension; Swiss Association against High Blood Pressure; U.S. National High Blood Pressure Education Program; Zaire League against Arterial Hypertension; and the International Green Cross. Requests for reprints or for permission to reproduce this article, or part of it, should be sent to the Secretary-General, World Hypertension League, 20 avenue du Bouchet, 1209 Geneva, Switzerland. A French translation of this article will appear in a later issue of the Bulletin.
}

\section{ADVANTAGES AND PROBLEMS}

\section{Physiological background: the value of home measurements}

Blood pressure is inherently variable and the first observations of measurement through a 24-hour period showed wide fluctuations of the order of $50 / 20 \mathrm{mmHg}(3)$. This variability was subsequently confirmed by intra-arterial readings during 24 hours (4-6), which showed that blood pressure changes rapidly with physical and mental activity (7) and falls substantially during sleep $(4,8)$. In addition to this spontaneous variability in pressure, the blood pressure in many subjects rises when they are in a doctor's office or clinic. For example, in a recent study it was shown that when a physician measured the blood pressure, the average rise in systolic and diastolic pressures was $14 \mathrm{mmHg}$ and $7 \mathrm{mmHg}$, respectively; there was very wide individual scatter, though some patients may have lower clinic than home readings (9). In a particular subject, therefore, the unpredictable rise in blood pressure in the doctor's clinic may present problems for both the diagnosis and the treatment of hypertension. Thus, it may be useful to complement clinic readings with home measurements.

The "alerting response" induced by a clinic visit wanes after repeated measurements and this is one reason for the well-known decline in blood pressure observed with repeated clinic visits $(10,11)$. However, the response rarely disappears completely and, in some cases, remains large.

It has long been believed that blood pressure initially goes through a labile phase before becoming established at a high level. This is not true. The opposite occurs. Lability increases as the blood pressure level rises, though less than when expressed as a percentage of the mean pressure; it also increases with increasing age $(9,12)$. Clinical physiologists have shown that blood pressure becomes more vari- 
able as the sensitivity of the baroreflexes declines in hypertensive subjects $(4,5)$ and, strangely, the variations in heart rate decline as the blood pressure variability increases $(7,13)$. However, when it is realized that the baroreflex influences the blood pressure in large measure by moderating cardiac function, it can be appreciated that, as the baroreflex sensitivity declines, the heart rate will increase and its variability will decrease.

Variability in blood pressure has limited clinical importance. The severity of blood pressure, as revealed by left ventricular hypertrophy and fundus changes, has been reported to be more closely related to the average level of ambulatory blood pressure recorded at home than to pressures measured in the clinic. Variability does not seem to be predictive (14). Though more data are needed, the morbidity and mortality rates due to hypertension have been reported to be better correlated with the average ambulatory blood pressure level than with the clinic blood pressure measurement (15). Left ventricular hypertrophy is also more closely related to raised home blood pressure levels than to the clinic readings (13, 16, 17). The present evidence, though not conclusive, suggests that readings taken in the home environment may be of greater value in clinical assessment than the office readings; the latter are often higher, the rise in blood pressure occurring when a patient visits the physician. The readings obtained at home also appear to be a reasonable guide to prognosis.

\section{Clinical value of self-measurement}

Self-measurement for the assessment of blood pressure is of clinical value in several ways.

Confirming the diagnosis of hypertension. Repeated measurement of blood pressure in the clinic shows that approximately $50 \%$ of patients with mildly elevated pressures ultimately become normotensive $(10,11)$. This can be achieved almost immediately by self-assessment at home and unnecessary treatment can be avoided. Following the established criteria, the upper limit of normal blood pressure for home readings may be taken as $140 / 90 \mathrm{mmHg}$ or $18.7 /$ $12.0 \mathrm{kPa}$ (this applies to casual measurements also). Of course, a blood pressure exceeding $140 / 90 \mathrm{mmHg}$ does not necessarily indicate that drug treatment is advised, but some intervention in life-style would certainly have to be recommended (18).

Assessing the effects of therapy. Measurements in the clinic may not accurately monitor blood pressure responses to therapy (19-21). The reliability and accuracy of home readings have three advantages. (1) The target pressure can be defined clearly; the aim should be a diastolic pressure of $<90 \mathrm{mmHg}$. (2) An excessive reduction in pressure, which may occur if one follows the clinic readings, can be avoided. (3) The interval between clinic visits can be made longer.

Self-measurement of blood pressure provides the possibility of an individually tailored drug treatment. It is complementary to drug studies; any placebo effect in self-measurement will become attenuated or even eliminated in the course of time, so that the blood-pressure-lowering effect of different kinds of treatment can be measured more easily and more reliably.

\section{Further advantages}

The availability of self-measurement may lead to the detection of an elevated blood pressure in persons previously unaware of the problem.

For individuals with borderline hypertension, the data collected may be helpful in documenting blood pressure patterns, which may assist in determining the proper treatment regimen. Self-measurement at home may encourage the patients and members of their family to participate more actively in the treatment programme. In some patients, the self-obtained evidence of a fall in blood pressure may encourage adherence to the prescribed treatment (i.e., improved compliance). The home readings may also aid the physician in modifying the treatment and in evaluating the effects of therapy; this could lead to simplification of treatment, including a reduction of drug dosage and fewer side-effects.

\section{Concerns about self-measurement}

On balance, the concerns about self-measurement of blood pressure must be weighed against the benefits. The problems include the possibility of inadequate training in measurement technique, consequent inaccurate readings, and misunderstanding of the observed values.

A single or even several self-measured elevated blood pressure readings are not an adequate basis for assuming that a person has hypertension. Elevated readings obtained in this way are only an indication that further evaluation is needed. The diagnosis of hypertension can only be made by a physician who interprets the significance of self-measured blood pressure readings along with other data relevant to the individual.

Repeated high readings due to a defective machine can lead an individual to a false self-diagnosis of "'high blood pressure." Users should be made aware of the possibility and implications of such measurements. Similarly, inaccurate low pressure readings also occur in a small proportion of persons.

Self-measurement may not suit some patients who become too concerned with their readings and alarmed by the normal fluctuations in pressure 
associated with daily living. Misinterpretation or overreaction to one or more measurements may cause psychological distress and even inappropriate selfadjustment of drug therapy. Such adjustment, without proper medical consultation, may lead to treatment errors or side-effects, or other problems.

It is indispensable that patients should be carefully instructed in the proper use of a blood-pressuremeasuring device and on the meaning of the results (see below). The apparatus has always to be used correctly and with care. In addition, the user should be highly motivated, but this is not always the case.

If the cuff is too large or too small for the subject's arm, false readings may result. When the arm circumference exceeds $36 \mathrm{~cm}$, the usual cuff may give falsely high values. Recommendations on the proper cuff size for a given arm girth are available (22), but have not been universally accepted. A hectic or noisy environment during measurement may also result in an excessively high reading.

\section{THE INSTRUMENTS}

Two classes of instruments used for the selfmeasurement of blood pressure will be discussed: portable devices for home measurement and stationary machines, usually installed in public places. A third class of instruments, ambulatory 24-hour bloodpressure monitors, require a high degree of the patient's cooperation and will not be discussed in this paper. These instruments are used mainly for investigative, sometimes diagnostic, purposes by specialist physicians and are not for self-measurement in the home.

\section{Home blood-pressure-measuring devices}

Several million families have already purchased a home blood-pressure-measuring device. These instruments may be a mercury sphygmomanometer, an aneroid manometer, or an electronic device.

The mercury sphygmomanometer is calibrated when manufactured and recalibration is unnecessary as long as the meniscus of the mercury column is exactly at 0 , when the cuff is deflated. If the mercury column is not at 0 or if it is dirty, the instrument should be returned to the manufacturer for cleaning and recalibration. The mercury sphygmomanometer is considered the most accurate and reliable device when properly used, and is the standard against which all other blood pressure instruments are compared.

Most instruments for self-measurement include a blood pressure cuff, an aneroid manometer and a stethoscope. These are expected to be reliable, accurate, and relatively inexpensive; to ensure consistent accuracy they should be calibrated at least once a year against a standard mercury sphygmomanometer using a Y-connector $(23,24)$.

The new digital readout (electronic) devices, which are the easiest of the three to use and usually do not require the use of a stethoscope, are more complex instruments. Many of them are fully automated; the push of a button inflates and deflates the cuff. While more expensive, these automated devices may be advantageous for those concerned about their ability to measure pressure accurately. However, because of their greater complexity and sensitivity, they are more likely to become inaccurate and should be recalibrated more frequently than once a year.

Caution: These instruments are highly sensitive. If the cuff with the sensor is improperly placed or if the arm moves during measurement, errors will occur. Patients should be advised to repeat the reading if unusual blood pressures are noted.

Digital readout (electronic) home blood-pressuremeasuring devices are being improved. Both instruments sensing the Korotkov sounds and those based on the oscillometric principle are, in most instances, sufficiently accurate for home use, if patients and the responsible health care professionals are aware of the potential problems.

\section{Stationary machines in public places}

The automated stationary machine, whether coinoperated or used free of charge, may be found at work sites, pharmacies, airports, and other public places. Evaluation of the commonly used instruments indicates that the readings can vary considerably between machines. The date of the last calibration of the instrument is the only measure of reliability and often this information is not provided for users. When compared with blood pressures taken by trained observers or by the random zero sphygmomanometer, machine-recorded blood pressure readings may vary by at least 5 to $10 \mathrm{mmHg}$ (both systolic and diastolic). The majority of errors are on the high side.

Blood pressure machines in public places should remind the public of the general significance of high blood pressure. On the other hand, there is much doubt about the validity of blood pressure values obtained in this way. As people are usually left on their own to use these machines, the instructions must be unequivocal and easy to execute. Regular calibrations should be carried out and the date clearly indicated on the machine.

People making use of these machines are often under stress owing to noise or physical exertion. Consequently, the values may be higher than clinic or home readings. This enhances the diagnostic sensitivity, but reduces its specificity. Overtreatment may hardly be detected by this procedure. In any case, the machine's print-out should sum up the conclusions to 
be drawn from the blood pressure values measured and provide the user with some sensible recommendations.

Users should be informed about the limited reliability of automated or semi-automated stationary machines and urged to seek medical advice before taking any action as a result of a blood pressure reading from one of them.

\section{TEACHING OF SELF-MEASUREMENT}

\section{Who should teach?}

Teaching of self-measurement of blood pressure should be regarded as a medical intervention which requires competence and takes time. It also requires a good relationship between the patient and the teacher; a good knowledge of the patient's personality is essential. Either a doctor or a qualified nurse can be the teacher.

\section{Who should be taught?}

Only patients likely to derive benefit from prolonged practice of self-measurement should be selected. The physician should carefully weigh the possible hazards of self-measurement for the patient, as he would do before any therapeutic intervention. Persons too concerned with their blood pressure levels, or disease in general, should be advised against self-measurement of blood pressure, even against their own wishes. In general, neurotic or anxious individuals are considered unsuitable. Nevertheless, some patients may cope better with their anxiety when given the opportunity of reassuring themselves by self-readings.

Patients or family members, whatever their social or intellectual background, may be selected, provided that they are sufficiently motivated, have the necessary sensory acuity, understand the objective, and are capable of learning the necessary skills. Elderly patients may find the learning of new skills more difficult than younger ones. Patients with physical handicaps, e.g., Parkinson's disease or syndrome, are obviously unsuitable for self-measurement.

\section{What to teach and how?}

The objectives the patient should achieve after being taught are:

(i) the correct and systematic taking of blood pressure readings;

(ii) periodic reporting to the treating physician.

The elementary notions to be understood by the patient include the following: (i) systolic and diastolic values;

(ii) heart (or pulse) rate;

(iii) variability of blood pressure and heart rate (no alarm if occasional values are high or low);

(iv) dependence of measured values on correct placement of the pick-up;

(v) functioning of the instrument;

(vi) self-measurement should not lead to self-medication.

The skills the patient should learn are as follows:

(i) taking at least five minutes rest before measurement;

(ii) placing the cuff in the proper position, with special attention to the position of the pick-up;

(iii) assuming a relaxed body posture and placing the arm and the instrument correctly;

(iv) inflating and deflating the cuff at the proper rates;

(v) in the auscultatory method, using the stethoscope and listening to the arterial sounds and identifying them;

(vi) coordinating the handling of the valve, listening to the sounds, and reading of the manometer figures;

(vii) reading the figures correctly;

(viii) counting the pulse rate (unless indicated by the instrument);

(ix) recording the values carefully and systematically;

(x) checking the instrument for visible shortcomings (e.g., dirty glass tube, faulty valve, leaking rubber tubes or joints).

Repeated teaching sessions are advisable. The patient should also be given written instructions, preferably an illustrated guide. With the auscultatory method, several simultaneous checks of the patient's reading are necessary using a Y-connector. The patient's ability to take the pressure correctly should be tested initially, and retested periodically, preferably at 6-month intervals.

\section{Support for the patient}

Self-measurement of blood pressure must be regarded as part of an integrated regimen. Besides the written instructions on how to use the machine and how to evaluate the readings, the patients should be given the possibility of contacting the physician or the nurse, if they feel insecure or alarmed. In case of breakdown of the instrument, especially the automated, electronic devices, the patient should have access to prompt servicing. When advising the patient which instrument to purchase, preference should be given to products for which servicing is readily available. Similarly, the patient should be assisted 
and, at least once a year, should be reminded (orally or by mail) to have the instrument checked.

The patient's self-recorded values should be discussed at regular intervals with the physician or nurse; such attention is valuable psychological support and complements the other measures of treatment and management.

\section{ACKNOWLEDGEMENTS}

This article includes extracts taken from Hunt, J. C., Frohlich, E. D., Moser, M., Roccella, E. J. \& Keighley, E. A. Devices used for self-measurement of blood pressure. Revised statement of the National High Blood Pressure Education Program. Archives of internal medicine, 145: 2231-2234 (1985). Permission to use the text is gratefully acknowledged. Passages are also quoted from two unpublished documents on self-measurement, prepared by J. Conway and $\mathrm{M}$. Anlauf; their collaboration is much appreciated.

\section{REFERENCES}

1. HUNT, J. C. ET AL. Devices used for self-measurement of blood pressure. Revised statement of the National High Blood Pressure Education Program. Archives of internal medicine, 145: 2231-2234 (1985).

2. DeUTSCHE LIGA ZUR BEKÄMPFUNG DES HOHEN BLUTDRUCKES. [Self-measurement of blood pressure.] 2nd ed. Heidelberg, 1984 (in German).

3. Richardson, D. W. ET AL. Changes in arterial pressure during sleep in man. Hypertension, 16: 62-75 (1967).

4. SMYTH, H. S. ET AL. Reflex regulation of arterial pressure during sleep in man. Circulation research, 24: 109-121 (1969).

5. BRistow, J. D. ET AL. Cardiovascular and respiratory changes during sleep in normal and hypertensive subjects. Cardiovascular research, 3: 476-485 (1969).

6. BEVAN, A. T. ET AL. Direct arterial pressure recording in unrestricted man. Clinical science, 36: 329-334 (1986).

7. CONWAY, J. ET AL. Neural and humoral mechanisms involved in blood pressure variability. Journal of hypertension, 2: 203-208 (1984).

8. DE LEEUW, P. W. ET AL. Effect of sleep on blood pressure and its correlates. Clinical and experimental hypertension, Part A, 7: 179-186 (1985).

9. NIELSEN, P. E. ET AL. Home readings of blood pressure in assessment of hypertensive patients. Acta medica Scandinavica, suppl. 714: 147-151 (1986).

10. Australian National Blood Pressure Study Management Committee. The Australian therapeutic trial in mild hypertension. Lancet, 1: 1261-1267 (1980).

11. CAREY, R. M. et AL. The Charlottesville blood pressure survey: value of repeated blood pressure measurements to determine the prevalence of labile and sustained hypertension. Journal of the American Medical Association, 236: 847-851 (1976).

12. MANCIA, G. ET AL. Arterial baroreflex and blood pressure and heart rate variabilities in humans. Hypertension, 8: 147-153 (1986).

13. LITTLER, W. A. ET AL. Continuous recording of direct arterial pressure and electrocardiogram in unrestricted man. British medical journal, 3: 76-78 (1972).

14. SOKOLOW, M. ET AL. Relationship between the level of blood pressure measured casually and by portable recorders and severity of complications in essential hypertension. Circulation, 34: 279-298 (1966).

15. PERloff, D. ET AL. The prognostic value of ambulatory blood pressure. Journal of the American Medical Association, 249: 2792-2798 (1983).

16. DEVEREUX, R. B. ET AL. Left ventricular hypertrophy in patients with hypertension: importance of blood pressure response to regularly recurring stress. Circulation, 68: 470-476 (1983).

17. DRAYER, J. I. M. ET AL. Blood pressure as a determinant of cardiac left ventricular muscle mass. Archives of internal medicine, 143: 90-92 (1983).

18. 1986 Guidelines for the treatment of mild hypertension: Memorandum from a WHO/ISH Meeting. Bulletin of the World Health Organization, 64: 31-35 (1986).

19. ANDERSEN, A. R. \& NIELSEN, P. E. Home readings of blood pressure in evaluation of hypertensive subjects using a new self-recording manometer. Acta medica Scandinavica, suppl. 670: 97-104 (1981).

20. Corcoran, A. C. ET AL. The evaluation of antihypertensive procedures, with particular reference to their effects on blood pressure. Annals of internal medicine, 43: 1161-1177 (1955).

21. FreIs, E. D. The discrepancy between home and office recordings of blood pressure in patients under treatment with pentapyrrolidinium; importance of home recordings in adjusting dosages. Medical annals of the District of Columbia, 23: 363-367 (1954).

22. MAXWELl, M. H. ET AL. Error in blood-pressure measurement due to incorrect cuff size in obese patients. Lancet, 2: 33-36 (1982).

23. HORAN, M. J. ET AL. Ambulatory blood pressure monitoring: recent advances and clinical applications. American heart journal, 101: 843-848 (1981).

24. AMERICAN NATIONAL Red Cross. The lowdown on high blood pressure. Instructor guide. Washington, DC, 1984. 